\title{
Impulsive Consensus Tracking of Multiagent Systems with Quantization and Input Delays Using Position-Only Information
}

\author{
Hong Zhou, Yu-Zhen Qin, Kuo Feng, Wen-Shan Hu, and Zhi-Wei Liu \\ Department of Automation, Wuhan University, Wuhan 430072, China \\ Correspondence should be addressed to Zhi-Wei Liu; liuzw@whu.edu.cn
}

Received 2 December 2013; Accepted 15 January 2014; Published 20 February 2014

Academic Editor: Huaicheng Yan

Copyright ( 2014 Hong Zhou et al. This is an open access article distributed under the Creative Commons Attribution License, which permits unrestricted use, distribution, and reproduction in any medium, provided the original work is properly cited.

\begin{abstract}
This paper investigates the consensus tracking problem for second-order multi-agent systems without/with input delays. Randomized quantization scheme is considered in the communication channels, and impulsive consensus tracking algorithms using position-only information are proposed for the consensus tracking of multi-agent systems. Based on the algebraic graph theory and stability theory of impulsive systems, sufficient and necessary conditions for consensus tracking are studied. It is found that consensus tracking for second-order multi-agent systems without/with input delays can be achieved by appropriately choosing the sampling period and control gains which are determined by second/third degree polynomials. Simulations are performed to validate the theoretical results.
\end{abstract}

\section{Introduction}

During the last two decades, the consensus problem in multiagent systems has attracted considerable attention due to its broad applications including synchronization [1], formation control [2], flocking [3], and sensor networks [4]. The main objective of consensus problem in multiagent systems is to design distributed control that enables all agents in a network to reach an agreement with a certain characteristic.

Recently, the leader-following structure containing one leader and some followers has been introduced into the consensus. Particularly, consensus with a static leader is named consensus regulation problem, and consensus with an active leader is named consensus tracking problem [5]. In the consensus tracking problem, a portion of the followers can obtain the leader's information while other followers can only obtain their neighbors' information. The task of consensus tracking is to make all the followers track the leader. Many valuable results on consensus tracking have been obtained in the existing literatures with different features including variable topology [6, 7], coupling time delays [8], and networkbased communication [9].

It is worthwhile to mention that most of the consensus tracking algorithms proposed in the above-mentioned literatures require both position and velocity information among neighbouring agents. Unfortunately, in order to reduce equipment costs and network traffic, the agents might not be equipped with velocity sensors in many applications such as some robots and air vehicles systems. Thus the velocity of each agent may not be obtained in these special multiagent systems. To address the limitation, consensus algorithms without any velocity information are proposed in [10-12].

On the other hand, in all of the aforementioned works, one major shortcoming of the proposed algorithms for the consensus tracking of multiagent systems is the reliance on the exchange of analog data. It demands quite a broad bandwidth and enough communication power in the information interaction. But in practical situations, communication channels are always with finite bandwidth and finite power so that only a finite number of bits can be transmitted [1315]. When the constraints are considered, communication with unquantized data is impractical $[16,17]$. The authors proposed distributed algorithms in which the agents utilize quantized communication information to communicate with each other $[14,18-20]$. The probabilistic quantization scheme was adopted in the agents interaction $[14,18]$. In [21], logarithmic quantizer was used to quantize the state of agents in the multiagent systems. 
Inspired by the above discussion, randomized quantization scheme is considered in the communication channels in this paper. At the sampling instant, the states of agents are sampled and quantized utilizing this scheme before being transmitted to their neighbors. Since the impulsive control strategy is proved to be a very effective control strategy in many fields due to its various advantages such as smaller control effort (only operates at sampling times), less information required (only needs the information at sampling times), and simple implementation [22-25], it is taken into consideration in our work. In conclusion, the main contribution of this paper includes the following two aspects: (1) the design of impulsive consensus tracking algorithms using the position-only information for the multiagent systems without/with input delays; (2) the introduction of randomized quantization scheme which is applied to the quantization of agents' information before communication, which is different from our previous works on impulsive algorithms $[22,24,26]$. It is worth pointing out that studying the effect of time delays on the consensus of multiagent systems is meaningful. Generally, there are two kinds of time delays in multiagent systems: communication delays and input delays. Communication delays are related to communication among agents while input delays are related to processing and connecting time for the packets arriving at each agent [27]. Input delay is studied in this paper. It is shown that the second-order multiagent systems without/with input delays can reach quantized consensus tracking if the sampling period and control gains are appropriately designed from polynomials with different orders.

The rest of the paper is organized as follows. In Section 2, preliminaries and problem formulation are given. The impulsive consensus tracking algorithms utilizing quantized communication for second-order multiagent systems without/with time delays are presented in Section 3 and the quantized consensus tracking is proved to be reached. In Section 4, numerical examples are given to illustrate the theoretical analysis. Conclusions are finally drawn in Section 5 .

\section{Preliminary and Problem Formulation}

In this section, some basic concepts used throughout this paper are introduced. Let $\mathbb{R}$ denote the set of real numbers and let $\mathbb{N}=\{1,2,3, \ldots\} .1_{n}=(1,1, \ldots, 1)^{T}$ is the column vector. $I_{n}$ is the identity matrix of order $n .0_{n \times m}$ denotes the matrix with all elements equal to zero.

2.1. Preliminaries in Graph Theory. For a multiagent system with $n$ agents, let $\mathscr{G}=\{\mathscr{V}, \mathscr{E}, \mathscr{A}\}$ be a directed graph with the set of vertices $\mathscr{V}(\mathscr{G})=\left\{v_{1}, v_{2}, \ldots, v_{n}\right\}$, the set of edges $\mathscr{E}(\mathscr{G}) \subseteq \mathscr{V}(\mathscr{G}) \times \mathscr{V}(\mathscr{G})$, and a weighted adjacency matrix $\mathscr{A}(\mathscr{G})=\left(a_{i j}\right)_{n \times n}$. An edge of $\mathscr{G}$ is denoted by $\theta_{i j}=\left(v_{j}, v_{i}\right)$, where $v_{j}$ is called the parent vertex of $v_{i}$ and $v_{i}$ the child of $v_{j}$. The adjacency elements associated with the edges are nonnegative. For $i, j \in \mathscr{V}, \theta_{i j} \in \mathscr{E}(\mathscr{G}) \Leftrightarrow a_{i j}>0$, and assume that $a_{i i}=0$. The set of neighbors of node $v_{i}$ is denoted by $\mathcal{N}_{i}=\left\{v_{j} \in \mathscr{V}(\mathscr{G}) \mid\left(v_{j}, v_{i}\right) \in \mathscr{E}(\mathscr{G}), j \neq i\right\}$. The Laplacian matrix $L=\left(l_{i j}\right)_{n \times n}$ is defined as $l_{i j}=\sum_{k=1, k \neq i}^{n} a_{i k}$, for $i=j$, and $l_{i j}=-a_{i j}$, for $i \neq j$, where $i, j=1,2, \ldots, n$.

2.2. Definitions and Notations. For a multiagent system, an agent is called a leader if the agent has no neighbor and an agent is called a follower if the agent has neighbors. In this paper, we will consider a multiagent system consisting of one active leader and $n$ followers. Let $\mathscr{G}$ denote the interaction topology of the followers and $L$ be Laplacian matrix of $\mathscr{G}$. Let $\widetilde{\mathscr{G}}$ denote the interaction topology of the multiagent system containing the followers and the leader. Let $B=\left(b_{i}\right)_{n \times n}$ describe the connection between follower $i$ and the leader. $b_{i}>0$ if and only if there exists a direct path from the leader to the follower; otherwise, $b_{i}=0$. The multiagent system with $n$ followers and one leader can be described by

$$
\begin{gathered}
\dot{\xi}_{0}(t)=\zeta_{0}(t), \\
\dot{\zeta}_{0}(t)=a(t), \\
\dot{\xi}_{i}(t)=\zeta_{i}(t), \\
\dot{\zeta}_{i}(t)=a(t)+u_{i}(t),
\end{gathered}
$$

where $\xi_{i}(t) \in \mathbb{R}$ and $\zeta_{i}(t) \in \mathbb{R}, i=1,2, \ldots, n$, are the position and velocity of agent $i$ and $u_{i}(t)$ is control input at time $t$. $\xi_{0}(t) \in \mathbb{R}, \zeta_{0}(t) \in \mathbb{R}$, and $a(t)$ are the position, velocity, and the acceleration of the dynamic leader, respectively. The followers aim to track the leader in this paper. It is assumed that the communication among agents only occurs at sampling instants, and the sampling intervals are periodic with period $h$. The sampling information needs to be quantized before being transmitted to the neighbor agents.

Randomized Quantization Scheme. In the following, a quantization scheme adopted in this paper is introduced. Suppose that the scalar value $\xi_{i}$ is quantized by a uniform quantizer with quantization interval $\delta$. Obviously, there exists an integer $k$ such that $\xi_{i} \in[k \delta,(k+1) \delta)$. Inspired by $[14,28]$, the data can be quantized by the probabilistic manner:

$$
\operatorname{Pr}\left\{\xi_{i}=k \delta\right\}=1-\chi, \quad \operatorname{Pr}\left\{\xi_{i}=(k+1) \delta\right\}=\chi,
$$

where $\chi=\left(\xi_{i}-k \delta\right) / \delta$. Define $\mathbb{Q}(\cdot)$ as the quantization operation. One has expectation and variance in the following form:

$$
E\left(\mathbb{Q}\left(\xi_{i}\right)\right)=\xi_{i}, \quad E\left(\left(\mathbb{Q}\left(\xi_{i}\right)-\xi_{i}\right)^{2}\right) \leq \frac{\delta^{2}}{4}
$$

In this scheme, the randomized quantized value is equal to the original unquantized value in expectation. Let $\varepsilon_{i}=$ $\mathbb{Q}\left(\xi_{i}\right)-\xi_{i}$ be the quantization error; the above formula can be rewritten as $E\left(\varepsilon_{i}^{2}\right) \leqslant \delta^{2} / 4$. 
Definition 1. The quantized consensus tracking in the multiagent system (1) is said to be achieved if, for any initial state,

$$
\begin{array}{r}
\lim _{t \rightarrow \infty} E\left(\left|\xi_{i}(t)-\xi_{0}(t)\right|^{2}\right) \leq p(\delta), \\
\lim _{t \rightarrow \infty} E\left(\left|\zeta_{i}(t)-\zeta_{0}(t)\right|^{2}\right) \leq q(\delta), \\
i=1,2, \ldots, n,
\end{array}
$$

where $p(\delta), q(\delta)$ are monotonously increasing functions satisfying $\lim _{\delta \rightarrow 0} p(\delta)=0, \lim _{\delta \rightarrow 0} q(\delta)=0$.

Before presenting the consensus tracking analysis, we first give the following lemmas which will be used in our work.

Lemma 2 (see [29]). $\mathscr{G}$ has a spanning tree if and only if L has a simple eigenvalue 0 together with the other eigenvalues whose real parts are all real ones. Therefore, $\widetilde{\mathscr{G}}$ contains a spanning tree if and only if all the eigenvalues of $L+B, \mu_{i}, i=1,2, \ldots, n$, have positive real parts.

Lemma 3 (see [30]). Consider a complex polynomial $R(s)=$ $s^{2}+(a+b i) s+c+d i$, where $a, b, c$, and d are real constants. Then $R(s)$ is Hurwitz stable if and only if $a>0$ and $a b d+a^{2} c-d^{2}>0$.

Lemma 4 (see [31]). Matrix $A$ and $\varepsilon>0$ are given. There exists a matrix norm \|\|$_{P}$ such that

$$
\rho(A) \leqslant\|A\|_{P} \leqslant \rho(A)+\varepsilon .
$$

\section{Consensus Tracking with Impulsive Algorithm}

3.1. Impulsive Algorithm Utilizing Quantization Communication without Input Delays. Assume sampling period in the multiagent system is a positive constant $h$, the sampling time sequence can be noted as a set $\Phi=\left\{0, t_{1}, \ldots, t_{k}, \ldots\right\}, k=$ $1,2, \ldots$. On count of the velocities $\zeta_{i}(t), \zeta_{0}(t), i=1,2, \ldots, n$, cannot be obtained; we propose the following impulsive algorithm for system (1):

$$
\begin{gathered}
\dot{\xi}_{i}(t)=\zeta_{i}(t), \\
\dot{\zeta}_{i}(t)=a(t)+u_{i}(t), \quad t \in\left(t_{k}, t_{k+1}\right], \\
\Delta \zeta_{i}(t)=-\beta_{1} \phi_{i}\left(t_{k}\right)-\beta_{2} \bar{z}_{i}(k), \\
z_{i}(k+1)=-\alpha_{1} \phi_{i}\left(t_{k}\right), \quad i=0,1,2, \ldots, n,
\end{gathered}
$$

where

$$
\begin{gathered}
\phi_{i}\left(t_{k}\right)=b_{i}\left(\xi_{i}\left(t_{k}\right)-\xi_{0}\left(t_{k}\right)\right)+\sum_{j \in N_{i}} l_{i j}\left(\xi_{j}\left(t_{k}\right)-\xi_{i}\left(t_{k}\right)\right), \\
\bar{z}_{i}(k)=b_{i} z_{i}(k)+\sum_{j \in N} l_{i j}\left(z_{j}(k)-z_{i}(k)\right),
\end{gathered}
$$

$u_{i}(t)=0, \Delta \zeta_{i}(t)=\zeta_{i}\left(t_{k}^{+}\right)-\zeta_{i}\left(t_{k}\right), \zeta_{i}\left(t_{k}^{+}\right)=\lim _{t \rightarrow t_{k}^{+}} \zeta_{i}(t), i=$ $1,2, \ldots, n$. What is worth pointing out is that $\xi_{i}\left(t_{k}\right)=p_{i}\left(t_{k}\right)+$ $\varepsilon_{i}\left(t_{k}\right)$, where $p_{i}\left(t_{k}\right)$ are the position information which are not quantized, and $\varepsilon_{i}\left(t_{k}\right)$ are denoted as the quantization error of agent $i$ at the time $t=t_{k}$ satisfying $E\left(\varepsilon_{i}^{2}\right) \leqslant \delta^{2} / 4$, where $\delta$ is the quantization interval of each agent in the system. Assume that $\zeta_{i}(t)$ is left-hand continuous at $t=t_{k} \cdot \alpha_{1}>0, \beta_{1}>0$, and $\beta_{2}>0$ are the control gains that need to be designed.

Denote $\zeta_{i}\left(t_{k}^{+}\right)=\zeta_{i}\left(t_{k+1}\right)$. From system (6), one can easily get

$$
\begin{gathered}
\xi_{i}\left(t_{k+1}\right)=\xi_{i}\left(t_{k}\right)+h \zeta_{i}\left(t_{k+1}\right), \\
\zeta_{i}\left(t_{k+1}\right)=\zeta_{i}\left(t_{k}\right)-\beta_{1} \phi_{i}\left(t_{k}\right)-\beta_{2} \bar{z}_{i}(k), \\
\phi_{i}\left(t_{k}\right)=b_{i}\left(\xi_{i}\left(t_{k}\right)-\xi_{0}\left(t_{k}\right)\right) \\
+\sum_{j \in \mathcal{N}_{i}} l_{i j}\left(\xi_{j}\left(t_{k}\right)-\xi_{i}\left(t_{k}\right)\right)+e_{i}(k),
\end{gathered}
$$

where $e_{i}(k)=b_{i}\left(\varepsilon_{i}\left(t_{k}\right)-\varepsilon_{0}\left(t_{k}\right)\right)+\sum_{j \in N_{i}} l_{i j}\left(\varepsilon_{j}\left(t_{k}\right)-\varepsilon_{i}\left(t_{k}\right)\right)$. Let $x_{i}(t)=\xi_{i}(t)-\xi_{0}(t), y_{i}(t)=\zeta_{i}(t)-\zeta_{0}(t)$, for $i=1,2, \ldots, n$. Assume that $y_{i}(t)$ is left-hand continuous at $t=t_{k}$. Let $y_{i}\left(t_{k}^{+}\right)=\lim _{t \rightarrow t_{k}^{+}} y_{i}(t)$. Then for Definition 1 , the quantized consensus tracking is achieved if and only if

$$
\lim _{t \rightarrow+\infty} E\left(\left|x_{i}(t)\right|^{2}\right) \leq p(\delta), \quad \lim _{t \rightarrow+\infty} E\left(\left|y_{i}(t)\right|^{2}\right) \leq q(\delta) .
$$

For $t \in\left(t_{k}, t_{k+1}\right]$, one has

$$
\begin{aligned}
y_{i}(t)= & y_{i}\left(t_{k}\right)-\beta_{1} b_{i} x_{i}\left(t_{k}\right) \\
& -\beta_{1} \sum_{j \in \mathcal{N}_{i}} l_{i j}\left(x_{j}\left(t_{k}\right)-x_{i}\left(t_{k}\right)\right)-\beta_{2} b_{i} z_{i}(k) \\
& -\beta_{2} \sum_{j \in \mathcal{N}} l_{i j}\left(z_{j}(k)-z_{i}(k)\right)-\beta_{1} e_{i}(k), \\
x_{i}(t)= & x_{i}\left(t_{k}\right)+\left(t-t_{k}\right) y_{i}\left(t_{k}\right)-\left(t-t_{k}\right) \beta_{1} b_{i} x_{i}\left(t_{k}\right) \\
& -\left(t-t_{k}\right) \beta_{1} \sum_{j \in \mathcal{N}_{i}} l_{i j}\left(x_{j}\left(t_{k}\right)-x_{i}\left(t_{k}\right)\right) \\
& -\left(t-t_{k}\right) \beta_{2} b_{i} z_{i}(k) \\
& -\left(t-t_{k}\right) \beta_{2} \sum_{j \in \mathcal{N}} l_{i j}\left(z_{j}(k)-z_{i}(k)\right) \\
& -\left(t-t_{k}\right) \beta_{1} e_{i}(k), \\
z_{i}(k+1)= & -\alpha_{1} b_{i} x_{i}\left(t_{k}\right) \\
& -\alpha_{1} \sum_{j \in \mathcal{N}_{i}} l_{i j}\left(x_{j}\left(t_{k}\right)-x_{i}\left(t_{k}\right)\right)-\alpha_{1} e_{i}(k) .
\end{aligned}
$$

Let $\Psi_{i}(k)=\left(x_{i}\left(t_{k}\right), y_{i}\left(t_{k}\right), z_{i}(k)\right)^{T}, \Phi(k)=\left(\Psi_{1}^{T}(k)\right.$, $\left.\Psi_{2}^{T}(k), \ldots, \Psi_{n}^{T}(k)\right)^{T}$, and $\Omega(k)=\left(e_{1}(k), e_{2}(k), \ldots, e_{n}(k)\right)^{T}$. Then controlled system (6) can be rewritten into a matrix form as

$$
\Phi(k+1)=F \Phi(k)+D \Omega(k)
$$


where $F=\left(I_{n} \otimes A-(L+B) \otimes C\right)$,

$$
\begin{gathered}
A=\left(\begin{array}{lll}
1 & h & 0 \\
0 & 1 & 0 \\
0 & 0 & 0
\end{array}\right), \quad C=\left(\begin{array}{ccc}
h \beta_{1} & 0 & h \beta_{2} \\
\beta_{1} & 0 & \beta_{2} \\
\alpha_{1} & 0 & 0
\end{array}\right), \\
D=-I_{n} \otimes\left(\begin{array}{c}
h \beta_{1} \\
\beta_{1} \\
\alpha_{1}
\end{array}\right) .
\end{gathered}
$$

Lemma 5. The controlled system (6) can achieve the quantized consensus tracking if and only if $\rho(F)<1$, where $\rho(\cdot)$ denotes the spectral radius of a matrix.

Proof. Define $\gamma(k)=E(\Phi(k) \otimes \Phi(k))$ and thus the quantized consensus tracking of system algorithm (6) can be studied by analyzing $\gamma(k)$ since

$$
E\left(\|\Phi(k)\|_{2}^{2}\right) \leq\|\gamma(k)\|_{1} \leq 3 n E\left(\|\Phi(k)\|_{2}^{2}\right),
$$

where $\|\cdot\|_{1},\|\cdot\|_{2}$ denote the 1-norm and 2-norm, respectively. Then from (11), one has

$$
\gamma(k+1)=(F \otimes F) \gamma(k)+(D \otimes D) E(\Omega(k) \otimes \Omega(k)) .
$$

Necessity. If $\rho(F) \geq 1$, the system (14) must be diverging and the system (6) cannot achieve the quantized consensus tracking since $\rho(F \otimes F) \geq 1$.

Sufficiency. If $\rho(F)<1$, then $\rho(F \otimes F)<1$. According to Lemma 4 , there exist a matrix norm $\|\cdot\|_{P}$ and a constant $\omega<$ 1 satisfying

$$
\|F \otimes F\|_{P}=\omega<\rho(F \otimes F)+\varepsilon<1,
$$

provided that $\varepsilon$ is small enough. And then from (14), one has

$$
\|\gamma(k+1)\|_{P} \leq \omega^{k+1}\|\gamma(0)\|_{P}+\sum_{i=0}^{k} \omega^{i} \widetilde{\delta},
$$

where $\widetilde{\delta}=12 n^{2}\|D \otimes D\|_{P} \delta, \delta$ is the quantized interval. It is easy to know that $\lim _{k \rightarrow \infty}\|\gamma(k+1)\|_{P} \leq \widetilde{\delta} /(1-\omega)$ since $\omega<$ 1. Therefore, there exists a positive constant $I \geqslant 0$ such that

$$
E\left(\|\Phi(k)\|_{2}^{2}\right) \leqslant \frac{I \widetilde{\delta}}{(1-\omega)} .
$$

Obviously, there exist constants $M>0$ and $N>0$ satisfying $\lim _{t \rightarrow \infty} E\left(\left\|x_{i}(t)\right\|_{2}^{2}\right) \leqslant M \widetilde{\delta} /(1-\omega), \lim _{t \rightarrow \infty} E\left(\left\|y_{i}(t)\right\|_{2}^{2}\right) \leqslant$ $N \widetilde{\delta} /(1-\omega)$; that is,

$$
\begin{gathered}
\lim _{t \rightarrow \infty} E\left(\left|\xi_{i}(t)-\xi_{0}(t)\right|^{2}\right) \leq M \delta, \\
\lim _{t \rightarrow \infty} E\left(\left|\zeta_{i}(t)-\zeta_{0}(t)\right|^{2}\right) \leq N \delta, \quad i=1,2, \ldots, n .
\end{gathered}
$$

According to Definition 1, it is easy to obtain that the quantized consensus tracking in the controlled system (6) can be achieved. Then this lemma is proved.
Theorem 6. The controlled system (6) can reach quantized consensus tracking if and only if the directed graph $\widetilde{G}$ has a spanning tree and the polynomial

$$
\begin{aligned}
g_{i}(\sigma)= & \left(h \beta_{1} \mu_{i}-h \alpha_{1} \mu_{i}^{2} \beta_{2}\right) \sigma^{2}+2 h \alpha_{1} \mu_{i}^{2} \beta_{2} \sigma \\
& +4-h \alpha_{1} \mu_{i}^{2} \beta_{2}-h \beta_{1} \mu_{i}
\end{aligned}
$$

is Hurwitz stable, where $\mu_{i}, i=1,2, \ldots, n$, are the eigenvalues of $L+B$.

Proof. Let

$$
F_{i}=\left(\begin{array}{ccc}
1-h \beta_{1} \mu_{i} & h & -h \beta_{2} \mu_{i} \\
-\beta_{1} \mu_{i} & 1 & -\beta_{2} \mu_{i} \\
-\alpha_{1} \mu_{i} & 0 & 0
\end{array}\right),
$$

where $\mu_{i}, i=1,2, \ldots, n$, are the eigenvalues of $L+B$. Assume that $L+B$ have $m$ different eigenvalues $u_{j}, j=1,2, \ldots, m$. There exists a invertible matrix $P$ such that $L+B=P^{-1} J P$, where $J=\operatorname{diag}\left(J_{1}, J_{2}, \ldots, J_{m}\right)$ and

$$
J_{j}=\left(\begin{array}{cccc}
\mu_{j} & 1 & 0 & 0 \\
0 & \mu_{j} & 1 & 0 \\
0 & 0 & \mu_{j} & 1 \\
0 & 0 & 0 & \mu_{j}
\end{array}\right)_{N_{j} \times N_{j}},
$$

where $N_{j}$ is the algebraic multiplicity of $\mu_{j}, j=1,2, \ldots, m$. Motivated by [30, 32], one has

$$
\begin{aligned}
X(k+1) & =\left(P \otimes I_{3}\right) F\left(P^{-1} \otimes I_{3}\right) X(k) \\
& \left(I_{n} \otimes A-J \otimes C\right) X(k)+H \Omega(k) \\
& =\left(\begin{array}{cccc}
\widetilde{J}_{j} & 0 & 0 & 0 \\
0 & \widetilde{J}_{j} & \cdots & 0 \\
\vdots & \vdots & \ddots & 0 \\
0 & 0 & 0 & \widetilde{J}_{j}
\end{array}\right) X(k)+H \Omega(k),
\end{aligned}
$$

where $\widetilde{J}_{j}=I_{n} \otimes A-J_{j} \otimes C, H=D$. According to Lemma 5, the quantized consensus tracking can be reached if and only if $\rho\left(\widetilde{J}_{j}\right)<1, j=1,2, \ldots, m$.

Note that

$$
\begin{aligned}
\widetilde{J}_{j} & =I_{n} \otimes A-J_{j} \otimes C \\
& =\left(\begin{array}{cccc}
A-\mu_{j} C & C & 0 & 0 \\
0 & A-\mu_{j} C & \ldots & 0 \\
0 & \ddots & \ddots & C \\
0 & 0 & 0 & A-\mu_{j} C
\end{array}\right), \\
& \left|\lambda I_{N_{j}}-\widetilde{J}_{j}\right| \\
& =\left(\begin{array}{cccc}
\lambda-A+\mu_{j} C & -C & 0 & 0 \\
0 & \lambda-A+\mu_{j} C & \cdots & 0 \\
0 & \ddots & \ddots & -C \\
0 & 0 & 0 & \lambda-A+\mu_{j} C
\end{array}\right) \\
& =\left|\lambda-A+\mu_{j} C\right|^{N_{j}} .
\end{aligned}
$$


This implies that $\rho\left(\widetilde{J}_{j}\right)<1, j=1,2, \ldots, m$ if and only if $\rho(A-$ $\left.\mu_{j} C\right)<1, j=1,2, \ldots, m$. As $F_{i}=A-\mu_{i} C$, the controlled system (6) can reach the quantized consensus tracking if and only if $\rho\left(F_{i}\right)<1, i=1,2, \ldots, n$. Note that

$$
\left|\lambda I_{3}-F_{i}\right|=\lambda^{3}+\left(h \beta_{1} \mu_{i}-2\right) \lambda^{2}+\left(1-h \alpha_{1} \mu_{i}^{2} \beta_{2}\right) \lambda .
$$

Let $f_{i}(\lambda)=\lambda^{2}+\left(h \beta_{1} \mu_{i}-2\right) \lambda+\left(1-h \alpha_{1} \mu_{i}^{2} \beta_{2}\right), i=1,2, \ldots, n$. $\rho\left(F_{i}\right)<1$ holds if and only if the polynomial $f_{i}(\lambda)$ is Schur stable.

Let

$$
\begin{aligned}
g_{i}(\sigma)= & (\sigma-1)^{2} f_{i}\left(\frac{\sigma+1}{\sigma-1}\right) \\
= & \left(h \beta_{1} \mu_{i}-h \alpha_{1} \mu_{i}^{2} \beta_{2}\right) \sigma^{2} \\
& +2 h \alpha_{1} \mu_{i}^{2} \beta_{2} \sigma+4-h \alpha_{1} \mu_{i}^{2} \beta_{2}-h \beta_{1} \mu_{i} .
\end{aligned}
$$

Obviously, the polynomial $f_{i}(\lambda)$ is Schur stable if and only if the polynomial $g_{i}(\sigma)$ is Hurwitz stable. And, then, the controlled system (6) can reach the quantized consensus tracking if and only if $g_{i}(\sigma)=\left(h \beta_{1} \mu_{i}-h \alpha_{1} \mu_{i}^{2} \beta_{2}\right) \sigma^{2}+$ $2 h \alpha_{1} \mu_{i}^{2} \beta_{2} \sigma+4-h \alpha_{1} \mu_{i}^{2} \beta_{2}-h \beta_{1} \mu_{i}$ is Hurwitz stable. Then Theorem 6 is proved.

Remark 7. In Theorem 6, a necessary and sufficient condition for the quantized consensus tracking for the controlled system (6) without input delays is established as a polynomial with order two. For a given topology, whether directed or undirected, one could choose appropriate control gains $\alpha_{1}, \beta_{1}, \beta_{2}$ and sampling period $h$ such that condition (19) is satisfied.

Theorem 8. Assume that $\mathscr{G}$ is a directed graph. The controlled system (6) can achieve the quantized consensus tracking if and only if the directed graph $\widetilde{\mathscr{G}}$ contains a spanning tree and

$$
\begin{gathered}
\operatorname{Re}\left(\mu_{i}\right)\left(\beta_{1}-\alpha \beta_{2} \operatorname{Re}\left(\mu_{i}\right)\right)-\operatorname{Im}^{2}\left(\mu_{i}\right) \alpha \beta_{2}>0 \\
a b d+a^{2} c-d^{2}>0
\end{gathered}
$$

holds, where

$$
\begin{gathered}
a=\operatorname{Re}\left(\frac{2 h \alpha_{1} \mu_{i} \beta_{2}}{\left(h \beta_{1}-h \alpha_{1} \mu_{i} \beta_{2}\right)}\right), \\
b=\operatorname{Im}\left(\frac{2 h \alpha_{1} \mu_{i} \beta_{2}}{\left(h \beta_{1}-h \alpha_{1} \mu_{i} \beta_{2}\right)}\right), \\
c=\operatorname{Re}\left(\frac{\left(4-h \alpha_{1} \mu_{i}^{2} \beta_{2}-h \beta_{1} \mu_{i}\right)}{\left(h \beta_{1} \mu_{i}-h \alpha_{1} \mu_{i}^{2} \beta_{2}\right)}\right), \\
d=\operatorname{Im}\left(\frac{\left(4-h \alpha_{1} \mu_{i}^{2} \beta_{2}-h \beta_{1} \mu_{i}\right)}{\left(h \beta_{1} \mu_{i}-h \alpha_{1} \mu_{i}^{2} \beta_{2}\right)}\right) .
\end{gathered}
$$

Proof. According to Theorem 6, note that

$$
\begin{aligned}
\widehat{g}_{i}(\sigma) & =\frac{g_{i}(\sigma)}{\left(h \beta_{1} \mu_{i}-h \alpha_{1} \mu_{i}^{2} \beta_{2}\right)} \\
& =\sigma^{2}+\frac{2 h \alpha_{1} \mu_{i} \beta_{2}}{\left(h \beta_{1}-h \alpha_{1} \mu_{i} \beta_{2}\right)} \sigma+\frac{4-h \alpha_{1} \mu_{i}^{2} \beta_{2}-h \beta_{1} \mu_{i}}{\left(h \beta_{1} \mu_{i}-h \alpha_{1} \mu_{i}^{2} \beta_{2}\right)} .
\end{aligned}
$$

From Lemma 2, if the directed graph $\widetilde{\mathscr{G}}$ has a spanning tree, then $\operatorname{Re}\left(\mu_{i}\right)>0, i=1,2, \ldots, n$. According to Lemma 3, the complex polynomial $\widehat{g}_{i}(\sigma)$ is Hurwitz stable if and only if $a>0$, and $a b d+a^{2} c-d^{2}>0$. It is easy to prove that $a>0$ is equivalent to $\operatorname{Re}\left(\mu_{i}\right)\left(\beta_{1}-\alpha \beta_{2} \operatorname{Re}\left(\mu_{i}\right)\right)-\operatorname{Im}^{2}\left(\mu_{i}\right) \alpha \beta_{2}>0$. Then this proof is completed.

Corollary 9. Assume that $\mathscr{G}$ is an undirected graph. The controlled system (6) can achieve the quantized consensus tracking if and only if the $\widetilde{\mathscr{G}}$ contains a spanning tree and

$$
\begin{gathered}
h<\frac{4}{\mu_{\max }\left(\beta_{1}+\alpha_{1} \mu_{\max } \beta_{2}\right)}, \\
\beta_{1}-\alpha_{1} \mu_{i} \beta_{2}>0
\end{gathered}
$$

holds, where $\mu_{\max }$ is the maximum eigenvalue of $L+B$.

Proof. According to Lemma 2, if the undirected graph $\widetilde{\mathscr{G}}$ has a spanning tree, then $\mu_{i}>0, i=1,2, \ldots, n$. As this corollary is a special case of Theorem 8 where $\operatorname{Re}\left(\mu_{i}\right)=\mu_{i}$ and $\operatorname{Im}\left(\mu_{i}\right)=0$, one can easily obtain the condition (29) by a simple calculation. Then Corollary 9 is proved.

3.2. Impulsive Algorithm Utilizing Quantization Communication with Input Delays. In some practical situations, the input time delays always exist, which cannot be ignored. When the time delays $\tau$ (assume that $\tau<h$ is time-invariant) are introduced into the protocol, one can consider the impulsive algorithm as

$$
\dot{\xi}_{i}(t)=\zeta_{i}(t),
$$

$$
\begin{gathered}
\dot{\zeta}_{i}(t)=a(t)+u_{i}(t), \quad t \in\left(t_{k}, t_{k+1},\right] \\
\Delta \zeta_{i}(t)=-\beta_{1} \phi_{i}\left(t_{k}\right)-\beta_{2} \bar{z}_{i}(k), \\
z_{i}(k+1)=-\alpha_{1} \phi_{i}\left(t_{k}\right), \quad i=0,1,2, \ldots, n,
\end{gathered}
$$

where

$$
\begin{aligned}
& \phi_{i}\left(t_{k}\right)= b_{i}\left(\xi_{i}\left(t_{k}-\tau\right)-\xi_{0}\left(t_{k}-\tau\right)\right) \\
&+\sum_{j \in N_{i}} l_{i j}\left(\xi_{j}\left(t_{k}-\tau\right)-\xi_{i}\left(t_{k}-\tau\right)\right), \\
& \bar{z}_{i}(k)=b_{i} z_{i}(k)+\sum_{j \in \mathcal{N}} l_{i j}\left(z_{j}(k)-z_{i}(k)\right) .
\end{aligned}
$$


Similar to the above analysis, it is easy to obtain that, for $t \in$ $\left(t_{k}, t_{k+1}\right]$,

$$
\begin{aligned}
y_{i}(t)= & y_{i}\left(t_{k}\right)-\beta_{1} b_{i} x_{i}\left(t_{k}-\tau\right) \\
& -\beta_{1} \sum_{j \in \mathcal{N}_{i}} l_{i j}\left(x_{j}\left(t_{k}-\tau\right)-x_{i}\left(t_{k}-\tau\right)\right)-\beta_{2} b_{i} z_{i}(k) \\
& -\beta_{2} \sum_{j \in \mathcal{N}} l_{i j}\left(z_{j}(k)-z_{i}(k)\right)-\beta_{1} e_{i}\left(t_{k}-\tau\right), \\
x_{i}(t)= & x_{i}\left(t_{k}\right)+h y_{i}\left(t_{k}\right)-h \beta_{1} b_{i} x_{i}\left(t_{k}-\tau\right) \\
& -h \beta_{1} \sum_{j \in \mathcal{N}_{i}} l_{i j}\left(x_{j}\left(t_{k}-\tau\right)-x_{i}\left(t_{k}-\tau\right)\right) \\
& -h \beta_{2} \sum_{j \in \mathcal{N}} l_{i j}\left(z_{j}(k)-z_{i}(k)\right)-h \beta_{1} e_{i}\left(t_{k}-\tau\right), \\
z_{i}(k+1)= & -\alpha_{1} b_{i} x_{i}\left(t_{k}-\tau\right) \\
& -\alpha_{1} \sum_{j \in \mathcal{N}_{i}} l_{i j}\left(x_{j}\left(t_{k}-\tau\right)-x_{i}\left(t_{k}-\tau\right)\right) \\
& -\alpha_{1} e_{i}\left(t_{k}-\tau\right),
\end{aligned}
$$

where

$$
\begin{aligned}
e_{i}\left(t_{k}-\tau\right)= & b_{i}\left(\varepsilon_{i}\left(t_{k}-\tau\right)-\varepsilon_{0}\left(t_{k}-\tau\right)\right) \\
& +\sum_{j \in \mathcal{N}_{i}} l_{i j}\left(\varepsilon_{j}\left(t_{k}-\tau\right)-\varepsilon_{i}\left(t_{k}-\tau\right)\right) .
\end{aligned}
$$

Let

$$
\begin{gathered}
\omega(k)=\left[e_{1}\left(t_{k}-\tau\right), e_{2}\left(t_{k}-\tau\right), \ldots, e_{3}\left(t_{k}-\tau\right)\right]^{T}, \\
\Xi(k)=\left[\Xi_{1}(k), \Xi_{2}(k), \ldots, \Xi_{n}(k)\right]^{T},
\end{gathered}
$$

where

$$
\Xi_{i}(k)=\left[x_{i}\left(t_{k}\right), y_{i}\left(t_{k}\right), x_{i}\left(t_{k}-\tau\right), y_{i}\left(t_{k}-\tau\right), z(k)\right]^{T}
$$

then the controlled system (30) can be rewritten as

$$
\Xi(k+1)=\widehat{F} \Xi(k)+\widehat{D} \omega(k),
$$

where $\widehat{F}=\left(I_{n} \otimes \widehat{A}-(L+B) \otimes \widehat{C}\right)$,

$$
\begin{gathered}
\widehat{A}=\left(\begin{array}{ccccc}
1 & h & 0 & 0 & 0 \\
0 & 1 & 0 & 0 & 0 \\
1 & h-\tau & 0 & 0 & 0 \\
0 & 1 & 0 & 0 & 0 \\
0 & 0 & 0 & 0 & 0
\end{array}\right), \\
\widehat{D}=I_{n} \otimes\left(\begin{array}{c}
-h \beta_{1} \\
-\beta_{1} \\
-(h-\tau) \beta_{1} \\
-\beta_{1}
\end{array}\right), \\
\widehat{C}=\left(\begin{array}{ccccc}
0 & 0 & h \beta_{1} & 0 & h \beta_{2} \\
0 & 0 & \beta_{1} & 0 & \beta_{2} \\
0 & 0 & (h-\tau) \beta_{1} & 0 & (h-\tau) \beta_{2} \\
0 & 0 & \beta_{1} & 0 & \beta_{2} \\
0 & 0 & \alpha_{1} & 0 & 0
\end{array}\right) .
\end{gathered}
$$

Lemma 10. When the input delays are considered, the controlled system (30) can achieve quantized consensus tracking if and only if $\rho(\widehat{F})<1$.

Proof. It is easy to prove this lemma in a way that is similar to what is used in Lemma 5. Define $\widehat{\gamma}(k)=E(\Xi(k) \otimes \Xi(k))$. Analogously, one has $\lim _{k \rightarrow \infty} E\left(\|\Xi(k)\|_{2}^{2}\right) \leqslant \widehat{I} \widetilde{\delta} /(1-\widehat{\omega})$, where $\widehat{\omega}=\|\widehat{F} \otimes \widehat{F}\|_{2}$. There exist positive constants $\widehat{M}, \widehat{N}>0$ such that $\lim _{k \rightarrow \infty} E\left(\left\|x_{i}\left(t_{k}\right)\right\|_{2}^{2}\right) \leqslant \widehat{M} \delta, \lim _{k \rightarrow \infty} E\left(\left\|y_{i}\left(t_{k}\right)\right\|_{2}^{2}\right) \leqslant \widehat{N} \delta$, so one has

$$
\lim _{t \rightarrow \infty} E\left(\left|\xi_{i}(t)-\xi_{0}(t)\right|^{2}\right) \leq \widehat{M} \delta
$$

$$
\lim _{t \rightarrow \infty} E\left(\left|\zeta_{i}(t)-\zeta_{0}(t)\right|^{2}\right) \leq \widehat{N} \delta, \quad i=1,2, \ldots, n .
$$

And then this proof is completed.

Theorem 11. The controlled (30) can achieve quantized consensus tracking if and only if the directed graph $\widetilde{\mathscr{G}}$ has a spanning tree and the polynomial

$$
\begin{aligned}
\widehat{g}_{i}(\sigma)= & \left(1+a_{2}+a_{1}+a_{0}\right) \sigma^{3}+\left(3+a_{2}-a_{1}-3 a_{0}\right) \sigma^{2} \\
& +\left(3-a_{2}-a_{1}+3 a_{0}\right) \sigma+\left(1-a_{2}+a_{1}-a_{0}\right)
\end{aligned}
$$

is Hurwitz stable, where $\mu_{i}, i=1,2, \ldots, n$, are the eigenvalues of $L+B, a_{2}=h \mu_{i} \beta_{1}-\tau \mu_{i} \beta_{1}-2, a_{1}=1+\tau \mu_{i} \beta_{1}-h \mu_{i}^{2} \alpha_{1} \beta_{2}+\tau \mu_{i}^{2} \alpha_{1} \beta_{2}$, $a_{0}=-\tau \mu_{i}^{2} \alpha_{1} \beta_{2}$.

Proof. Let

$$
\widehat{F}_{i}=\left(\begin{array}{ccccc}
1 & h & -\mu_{i} h \beta_{1} & 0 & -\mu_{i} h \beta_{2} \\
0 & 1 & -\mu_{i} \beta_{1} & 0 & -\mu_{i} \beta_{2} \\
1 & h-\tau & -\mu_{i}(h-\tau) \beta_{1} & 0 & -\mu_{i}(h-\tau) \beta_{2} \\
0 & 1 & -\mu_{i} \beta_{1} & 0 & -\mu_{i} \beta_{2} \\
0 & 0 & -\mu_{i} \alpha_{1} & 0 & 0
\end{array}\right),
$$

where $\mu_{i}, i=1,2, \ldots, n$ are the eigenvalues of $L+B$. According to Lemma 10, it is easy to know that the controlled system 
(30) can achieve quantized consensus tracking if and only if $\rho\left(\widehat{F}_{i}\right)<1, i=1,2, \ldots, n$. Note that

$$
\begin{aligned}
& \left|\lambda I_{5}-\widehat{F}_{i}\right| \\
& =\lambda^{2}\left(\lambda^{3}+\left(h \mu_{i} \beta_{1}-\tau \mu_{i} \beta_{1}-2\right) \lambda^{2}\right. \\
& \left.+\left(1+\tau \mu_{i} \beta_{1}-h \mu_{i}^{2} \alpha_{1} \beta_{2}+\tau \mu_{i}^{2} \alpha_{1} \beta_{2}\right) \lambda-\tau \mu_{i}^{2} \alpha_{1} \beta_{2}\right) .
\end{aligned}
$$

Let

$$
\begin{gathered}
\widehat{f}_{i}(\lambda)=\lambda^{3}+\left(h \mu_{i} \beta_{1}-\tau \mu_{i} \beta_{1}-2\right) \lambda^{2} \\
+\left(1+\tau \mu_{i} \beta_{1}-h \mu_{i}^{2} \alpha_{1} \beta_{2}+\tau \mu_{i}^{2} \alpha_{1} \beta_{2}\right) \lambda \\
-\tau \mu_{i}^{2} \alpha_{1} \beta_{2}, \\
a_{2}=h \mu_{i} \beta_{1}-\tau \mu_{i} \beta_{1}-2, \\
a_{1}=1+\tau \mu_{i} \beta_{1}-h \mu_{i}^{2} \alpha_{1} \beta_{2}+\tau \mu_{i}^{2} \alpha_{1} \beta_{2}, \quad a_{0}=-\tau \mu_{i}^{2} \alpha_{1} \beta_{2},
\end{gathered}
$$

and then one has $\widehat{f}_{i}(\lambda)=\lambda^{3}+a_{2} \lambda^{2}+a_{1} \lambda+a_{0}$. Let

$$
\widehat{g}_{i}(\sigma)=(\sigma-1)^{3} f\left(\frac{(\sigma+1)}{(\sigma-1)}\right) \text {. }
$$

It can be rewritten in the following form:

$$
\begin{aligned}
\widehat{g}_{i}(\sigma)= & \left(1+a_{2}+a_{1}+a_{0}\right) \sigma^{3}+\left(3+a_{2}-a_{1}-3 a_{0}\right) \sigma^{2} \\
& +\left(3-a_{2}-a_{1}+3 a_{0}\right) \sigma+\left(1-a_{2}+a_{1}-a_{0}\right) .
\end{aligned}
$$

Obviously, polynomial $\hat{f}_{i}(\lambda)$ is Schur stable if and only if polynomial $\widehat{g}_{i}(\sigma)$ is Hurwitz stable. It is easy to conclude that the controlled system (30) can achieve the quantized consensus tracking if and only if $\widehat{g}_{i}(\sigma)$ is Hurwitz stable. Then this theorem is proved.

Remark 12. Similar to Theorem 6, a necessary and sufficient condition for the quantized consensus tracking for the controlled system (30) is derived as a polynomial with order three in Theorem 11. For a given topology with determinate input delays $\tau$, whether directed or undirected, one could also choose appropriate control gains $\alpha_{1}, \beta_{1}, \beta_{2}$ and sampling period $h$ such that condition (39) is satisfied.

Corollary 13. Assume that the $\mathscr{G}$ is an undirected graph. The controlled system (30) can achieve quantized consensus tracking if and only if the $\widetilde{\mathscr{G}}$ consists of a spanning tree and

$$
\begin{gathered}
\beta_{1}>\mu_{i} \alpha_{1} \beta_{2}, \\
(h-2 \tau) \beta_{1}+(h+2 \tau) \mu_{i} \alpha_{1} \beta_{2}>0, \\
4-h \mu_{i} \beta_{1}+(h-4 \tau) \mu_{i}^{2} \alpha_{1} \beta_{2}>0, \\
4+(2 \tau-h) \mu_{i} \beta_{1}+(2 \tau-h) \mu_{i}^{2} \alpha_{1} \beta_{2}>0, \\
1+a_{2} a_{0}-a_{0}^{2}-a_{1}>0
\end{gathered}
$$

hold.

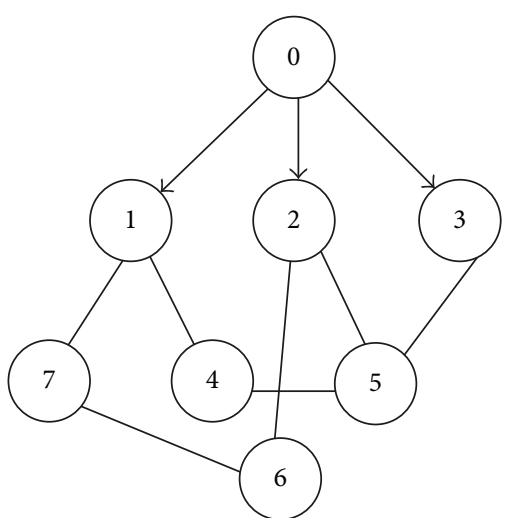

FIGURE 1: The interaction graph.

Proof. While the $\widetilde{\mathscr{G}}$ is the undirected graph, $\mu_{i}, i=1,2, \ldots, n$ are all real numbers. Thus $a_{2}, a_{1}, a_{0}$ are all real numbers too. According to Routh's stability criterion, $\widehat{g}_{i}(\sigma)$ is stable if and only if $1+a_{2}+a_{1}+a_{0}>0,3+a_{2}-a_{1}-3 a_{0}>0,3-a_{2}-$ $a_{1}+3 a_{0}>0,1-a_{2}+a_{1}-a_{0}>0$, and $\left(3-a_{2}-a_{1}+3 a_{0}\right)(3+$ $\left.a_{2}-a_{1}-3 a_{0}\right)-\left(1+a_{2}+a_{1}+a_{0}\right)\left(1-a_{2}+a_{1}-a_{0}\right)>0$. By solving the first four polynomials, one obtains condition (45). The fifth polynomial can be rewritten as condition (46). Therefore, quantized consensus tracking in controlled system (30) can be reached. This proof is completed.

\section{Numerical Simulations}

In this section, some numerical simulations are given to demonstrate the theorem analysis. Consider a multiagent system with 8 agents (a leader and 7 followers) which is shown in Figure 1. The Laplacian matrix $L$ is

$$
L=\left(\begin{array}{ccccccc}
2 & 0 & 0 & -1 & 0 & 0 & -1 \\
0 & 2 & 0 & 0 & -1 & -1 & 0 \\
0 & 0 & 1 & 0 & -1 & 0 & 0 \\
-1 & 0 & 0 & 2 & -1 & 0 & 0 \\
0 & -1 & -1 & -1 & 3 & 0 & 0 \\
0 & -1 & 0 & 0 & 0 & 2 & -1 \\
-1 & 0 & 0 & 0 & 0 & -1 & 2
\end{array}\right)
$$

where $B=\operatorname{diag}(1,1,1,0,0,0,0), b=(1,1,1,0,0,0,0)^{T}$, and $a(t)=0.1 \sin (t / 10)$. Choose quantized interval $\delta=0.2$. By simple calculation of $L$, one has $\mu_{\max }=4.7384$. Choose $\alpha_{1}=0.2, \beta_{2}=1$, and $\beta_{1}=1.2$. According to Theorem 8 , the quantized consensus tracking can be reached if and only if $h<$ 0.3931 . Let $h=0.39$, as it is shown in Figure 2, the quantized tracking consensus can be achieved. While $h=0.40$, it cannot be achieved, which is shown in Figure 3.

When input delays are taken into consideration, choose $\alpha_{1}=0.2, \beta_{1}=1, \beta_{2}=1, h=0.4$, and $\tau=0.02$, which satisfies Theorem 11. As it is shown in Figure 4, the quantized consensus tracking can be achieved. But when $\alpha_{1}=0.2, \beta_{1}=$ $1, \beta_{2}=1, h=0.4$, and $\tau=0.06$, the system cannot achieve the consensus tracking, which is shown in Figure 5.

Remark 14. As a matter of fact, the quantized consensus tracking is clearly not a strict consensus; that is, all agents 


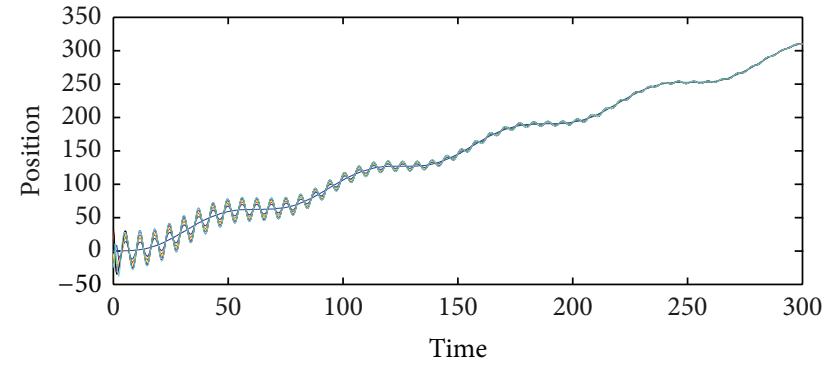

(a)

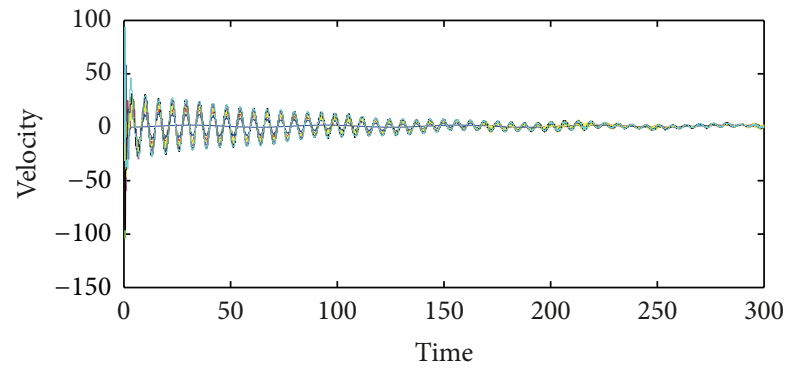

(b)

Figure 2: Position (a) and velocity (b) states of agents, where $h=0.39$ and $\delta=0.2$.

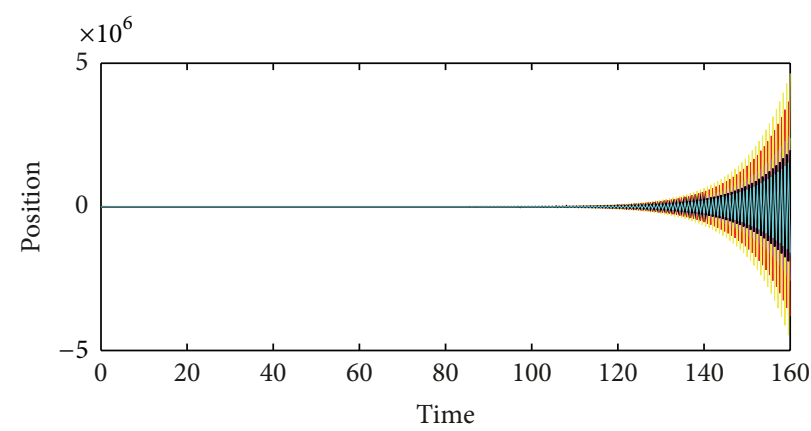

(a)

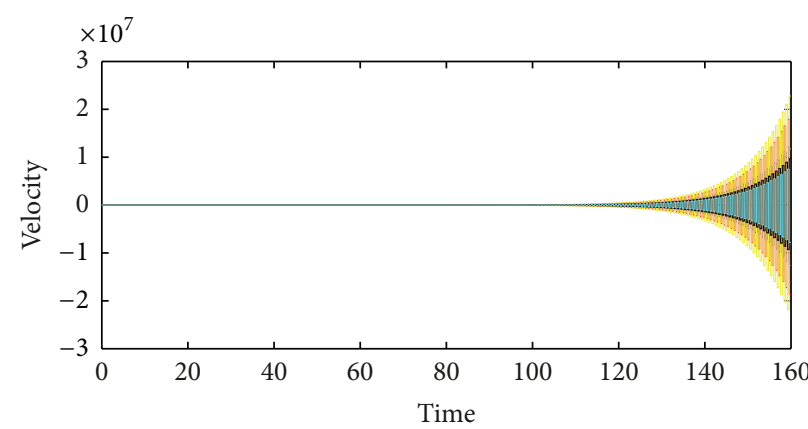

(b)

Figure 3: Position (a) and velocity (b) states of agents, where $h=0.40$ and $\delta=0.2$.

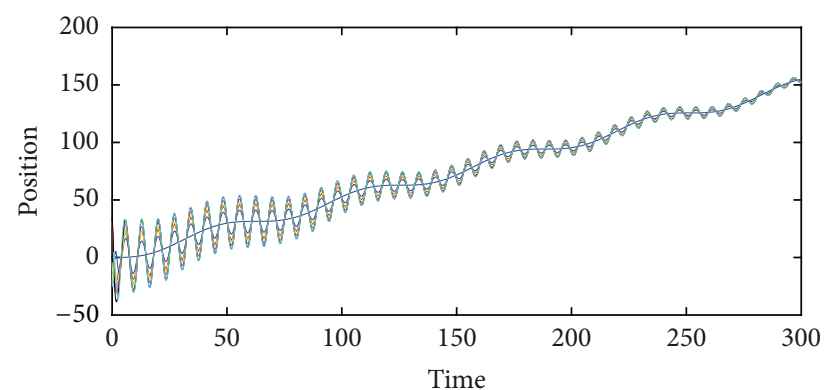

(a)

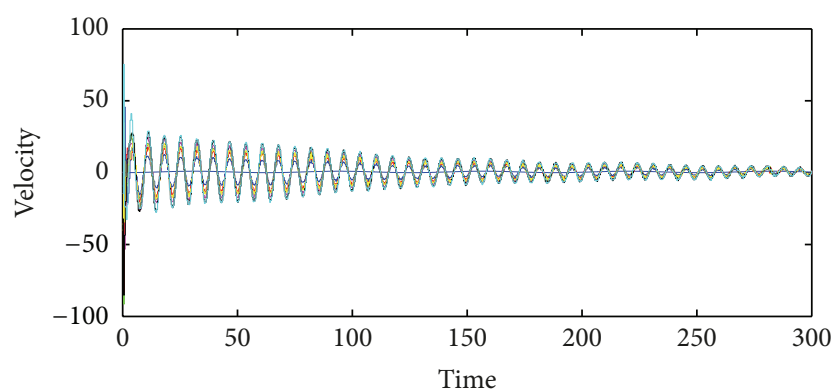

(b)

Figure 4: Position (a) and velocity (b) states of agents, where $h=0.4, \tau=0.02$, and $\delta=0.2$.

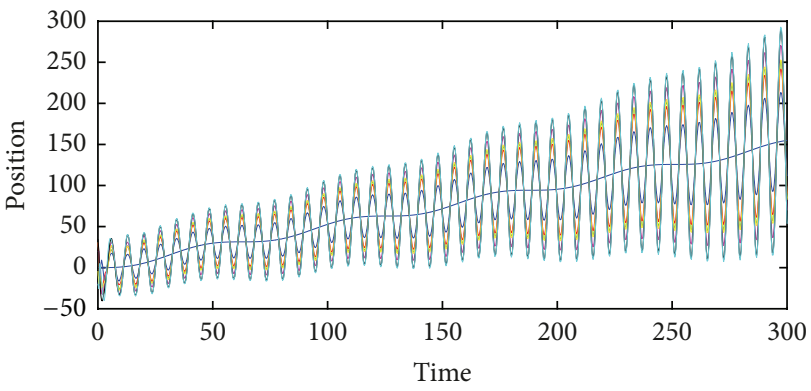

(a)

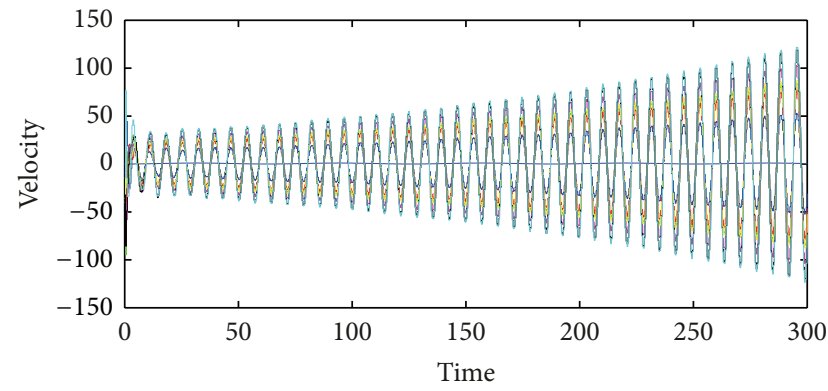

(b)

FIgURE 5: Position (a) and velocity (b) states of agents, where $h=0.4, \tau=0.06$, and $\delta=0.2$. 


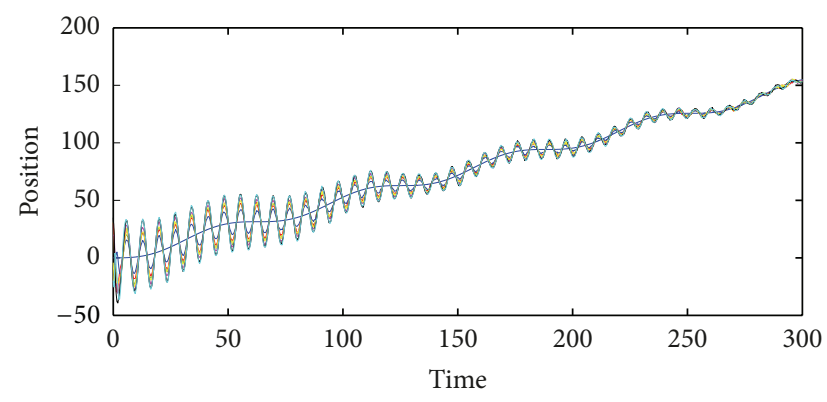

(a)

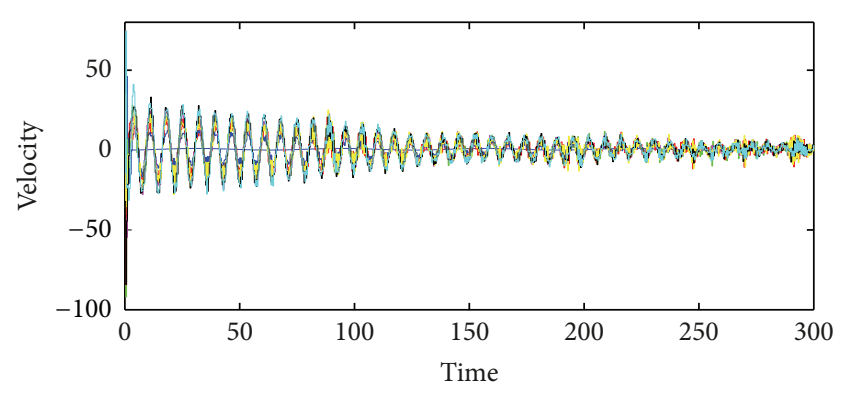

(b)

FIGURE 6: Position (a) and velocity (b) states of agents, where $h=0.4, \tau=0.02$, and $\delta=1$.

in the network do not have the same value as the leader. There are still some errors in quantized consensus tracking which can be found in the simulation results of Figures 4 and 6. According to Definition 1, this phenomenon is easily explained because the consensus quality is closely related to the quantized interval $\delta$ or even determined by it. Comparing Figure 4 with Figure 6 , it can be found that the later errors $(\delta=1)$ are bigger than the former $(\delta=0.2)$ under the same control gains, sampling interval, and time delay. Therefore, in order to achieve a more accurate quantized consensus tracking, smaller quantized interval should be taken into account in practical applications.

\section{Conclusions}

In this paper, the consensus tracking problems for secondorder multiagent systems without/with input delays are studied. We propose impulsive consensus tracking algorithms without velocity measurements. Considering the constraints of communication channels with finite bandwidth and finite power, randomized quantization scheme is introduced in our work. Information of agents in the multiagent systems is quantized using this scheme before being transmitted to their neighbors. Some sufficient and necessary conditions for consensus tracking are obtained. By appropriately choosing the sampling period and control gains such that this condition holds, the second-order multiagent systems without/with input delays can achieve quantized consensus tracking. Finally, numerical simulations are given to illustrate the theoretical analysis. Furthermore, future efforts will focus on adaptive and output feedback extensions of the developed controllers to reduce the required amount of state and model knowledge.

\section{Conflict of Interests}

The authors declare that there is no conflict of interests regarding the publication of this paper.

\section{Acknowledgments}

This work was supported in part by the National Natural Science Foundation of China (61304152, 61374064, and
61364017), National Key Technology Research and Development Program of the Ministry of Science and Technology of China (2013BAA01B01), and the China Postdoctoral Science Foundation funded project (2013T60738).

\section{References}

[1] Z. Li, Z. Duan, G. Chen, and L. Huang, "Consensus of multiagent systems and synchronization of complex networks: a unified viewpoint," IEEE Transactions on Circuits and Systems, vol. 57, no. 1, pp. 213-224, 2010.

[2] Z. Lin, B. Francis, and M. Maggiore, "Necessary and sufficient graphical conditions for formation control of unicycles," IEEE Transactions on Automatic Control, vol. 50, no. 1, pp. 121-127, 2005.

[3] R. Olfati-Saber, "Flocking for multi-agent dynamic systems: algorithms and theory," IEEE Transactions on Automatic Control, vol. 51, no. 3, pp. 401-420, 2006.

[4] R. Olfati-Saber and J. S. Shamma, "Consensus filters for sensor networks and distributed sensor fusion," in Proceedings of the 44th IEEE Conference on Decision and Control, and the European Control Conference (CDC-ECC '05), pp. 6698-6703, Seville, Spain, December 2005.

[5] Z. Meng, W. Ren, Y. Cao, and Z. You, "Leaderless and leaderfollowing consensus with communication and input delays under a directed network topology," IEEE Transactions on Systems, Man, and Cybernetics B, vol. 41, no. 1, pp. 75-88, 2011.

[6] Y. Hong, J. Hu, and L. Gao, "Tracking control for multiagent consensus with an active leader and variable topology," Automatica, vol. 42, no. 7, pp. 1177-1182, 2006.

[7] W. Ni and D. Cheng, "Leader-following consensus of multiagent systems under fixed and switching topologies," Systems \& Control Letters, vol. 59, no. 3-4, pp. 209-217, 2010.

[8] J. Hu and Y. Hong, "Leader-following coordination of multiagent systems with coupling time delays," Physica A, vol. 374, no. 2, pp. 853-863, 2007.

[9] L. Ding, Q.-L. Han, and G. Guo, "Network-based leaderfollowing consensus for distributed multi-agent systems," Automatica, vol. 49, no. 7, pp. 2281-2286, 2013.

[10] H. Su, X. Wang, and G. Chen, "A connectivity-preserving flocking algorithm for multi-agent systems based only on position measurements," International Journal of Control, vol. 82, no. 7, pp. 1334-1343, 2009.

[11] J. Mei, W. Ren, and G. Ma, "Distributed coordination for second-order multi-agent systems with nonlinear dynamics 
using only relative position measurements," Automatica, vol. 49, no. 5, pp. 1419-1427, 2013.

[12] Z.-W. Liu, Z.-H. Guan, X. Shen, and G. Feng, "Consensus of multi-agent networks with aperiodic sampled communication via impulsive algorithms using position-only measurements," IEEE Transactions on Automatic Control, vol. 57, no. 10, pp. 2639-2643, 2012.

[13] A. Kashyap, T. Başar, and R. Srikant, "Quantized consensus," Automatica, vol. 43, no. 7, pp. 1192-1203, 2007.

[14] T. C. Aysal, M. Coates, and M. Rabbat, "Distributed average consensus using probabilistic quantization," in Proceedings of the 14th IEEE/SP WorkShop on Statistical Signal Processing (SSP '07), pp. 640-644, Madison, Wis, USA, August 2007.

[15] H. Yan, Z. Su, H. Zhang, and F. Yang, "Observer-based $H_{\infty}$ control for discrete-time stochastic systems with quantisation and random communication delays," IET Control Theory \& Applications, vol. 7, no. 3, pp. 372-379, 2013.

[16] H. Zhang, H. Yan, F. Yang, and Q. Chen, "Quantized control design for impulsive fuzzy networked systems," IEEE Transactions on Fuzzy Systems, vol. 19, no. 6, pp. 1153-1162, 2011.

[17] Z.-H. Guan, C. Meng, R.-Q. Liao, and D.-X. Zhang, "Consensus of second-order multi-agent dynamic systems with quantized data," Physics Letters A, vol. 376, no. 4, pp. 387-393, 2012.

[18] T. C. Aysal, M. J. Coates, and M. G. Rabbat, "Rates of convergence for distributed average consensus with probabilistic quantization," in Proceedings of the Allerton Conference on Communication, Control, and Computing, Urbana, Ill, USA, September 2007.

[19] Z.-W. Liu, Z.-H. Guan, T. Li, X.-H. Zhang, and J.-W. Xiao, "Quantized consensus of multi-agent systems via broadcast gossip algorithms," Asian Journal of Control, vol. 14, no. 6, pp. 1634-1642, 2012.

[20] T. C. Aysal, M. J. Coates, and M. G. Rabbat, "Distributed average consensus with dithered quantization," IEEE Transactions on Signal Processing, vol. 56, no. 10, pp. 4905-4918, 2008.

[21] D. V. Dimarogonas and K. H. Johansson, "Stability analysis for multi-agent systems using the incidence matrix: quantized communication and formation control," Automatica, vol. 46, no. 4, pp. 695-700, 2010.

[22] Z.-H. Guan, Z.-W. Liu, G. Feng, and M. Jian, "Impulsive consensus algorithms for second-order multi-agent networks with sampled information," Automatica, vol. 48, no. 7, pp. 13971404, 2012.

[23] H. Zhang, H. Yan, T. Liu, and Q. Chen, "Fuzzy controller design for nonlinear impulsive fuzzy systems with time delay," IEEE Transactions on Fuzzy Systems, vol. 19, no. 5, pp. 844-856, 2011.

[24] Z.-W. Liu, H. Zhou, Z.-H. Guan, W.-S. Hu, L. Ding, and W. Wang, "Distributed impulsive consensus of the multiagent system without velocity measurement," Abstract and Applied Analysis, vol. 2013, Article ID 825307, 8 pages, 2013.

[25] H. Zhang, Z.-H. Guan, and G. Feng, "Reliable dissipative control for stochastic impulsive systems," Automatica, vol. 44, no. 4, pp. 1004-1010, 2008.

[26] Z.-H. Guan, Z.-W. Liu, G. Feng, and Y.-W. Wang, "Synchronization of complex dynamical networks with time-varying delays via impulsive distributed control," IEEE Transactions on Circuits and Systems, vol. 57, no. 8, pp. 2182-2195, 2010.

[27] Y.-P. Tian and C.-L. Liu, "Consensus of multi-agent systems with diverse input and communication delays," IEEE Transactions on Automatic Control, vol. 53, no. 9, pp. 2122-2128, 2008.
[28] Y. Zhang and Y.-P. Tian, "Consensus of data-sampled multiagent systems with random communication delay and packet loss," IEEE Transactions on Automatic Control, vol. 55, no. 4, pp. 939-943, 2010.

[29] F. Bullo, J. Cortés, and S. Martínez, Distributed Control of Robotic Networks: A Mathematical Approach to Motion Coordination Algorithms, Princeton University Press, Princeton, NJ, USA, 2009.

[30] W. Yu, W. X. Zheng, G. Chen, W. Ren, and J. Cao, "Second-order consensus in multi-agent dynamical systems with sampled position data," Automatica, vol. 47, no. 7, pp. 1496-1503, 2011.

[31] R. A. Horn and C. R. Johnson, Matrix Analysis, Cambridge University Press, Cambridge, UK, 2012.

[32] W. Yu, L. Zhou, X. Yu, J. Lu, and R. Lu, "Consensus in multiagent systems with second-order dynamics and sampled data," IEEE Transactions on Industrial Informatics, vol. 9, no. 4, pp. 2137-2146, 2012. 


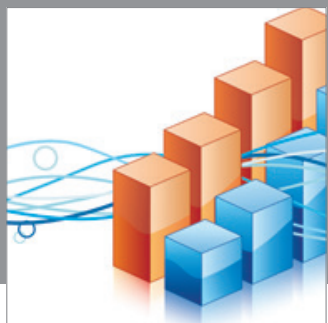

Advances in

Operations Research

mansans

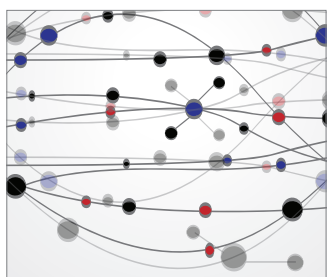

The Scientific World Journal
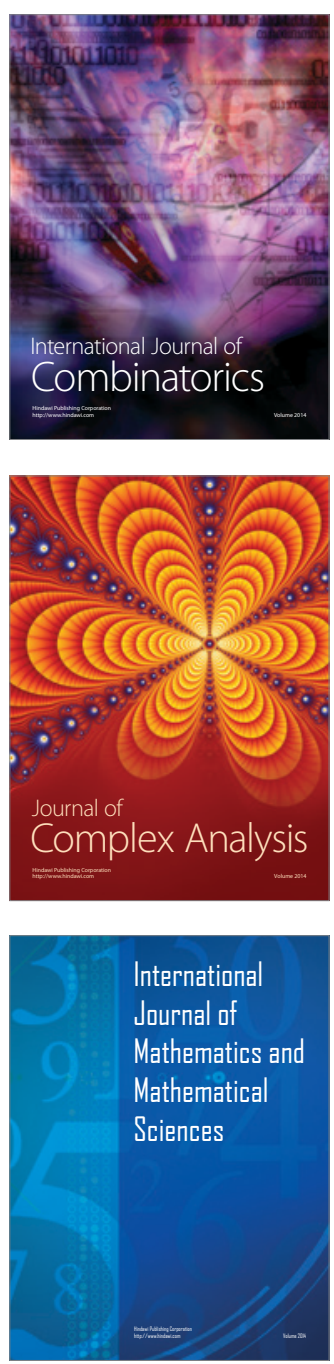
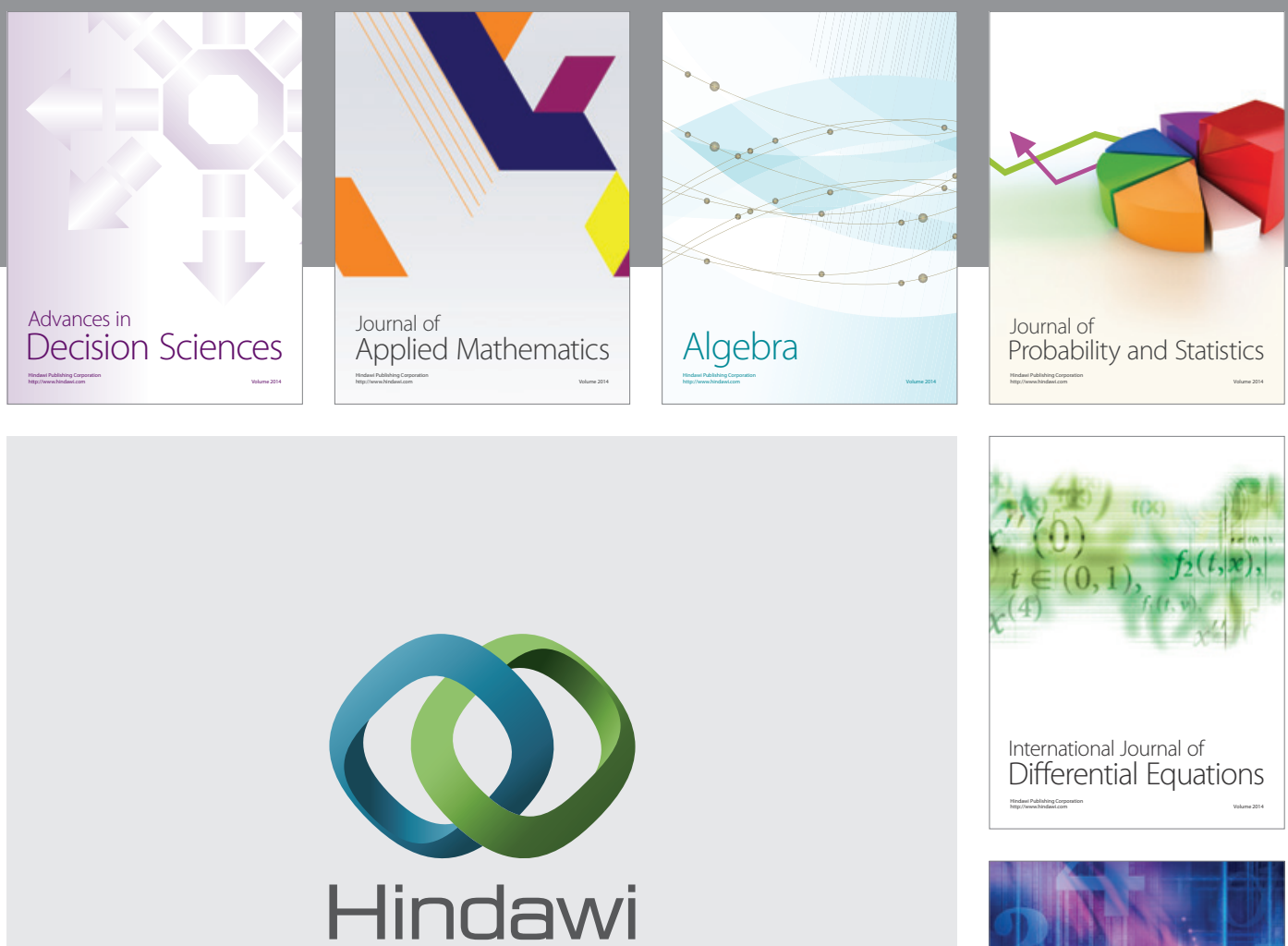

Submit your manuscripts at http://www.hindawi.com
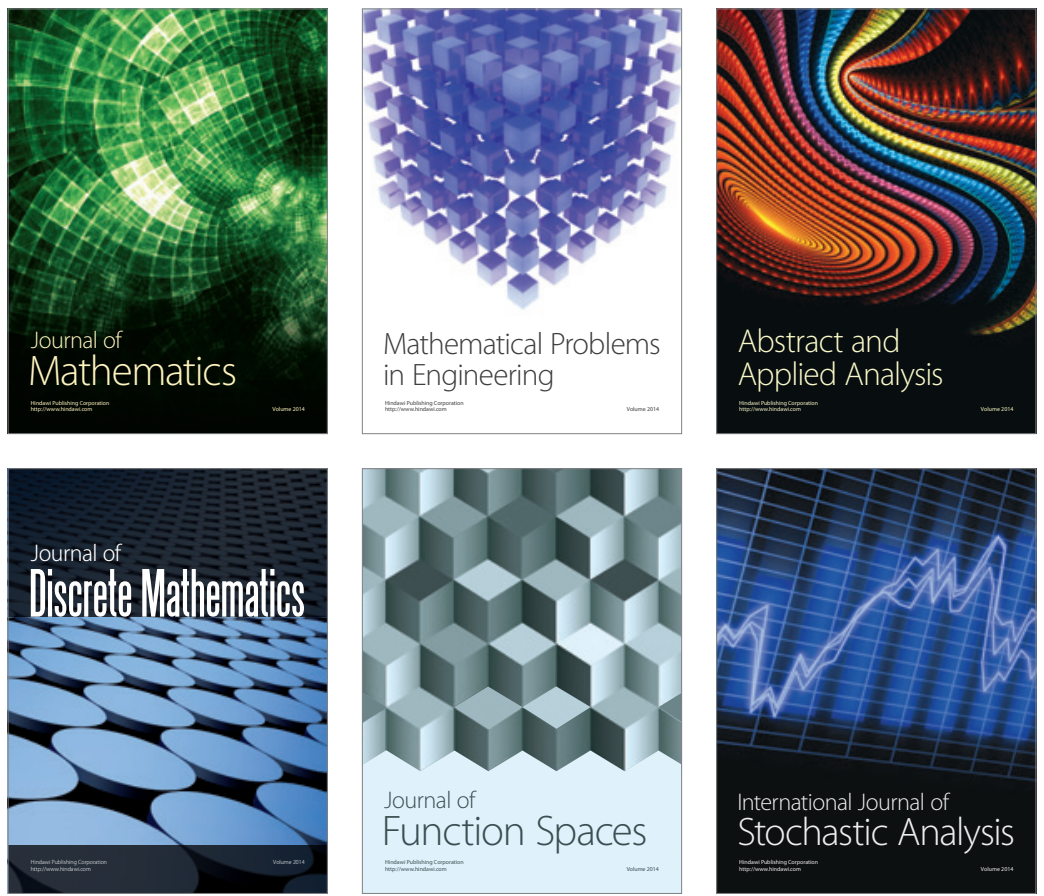

Journal of

Function Spaces

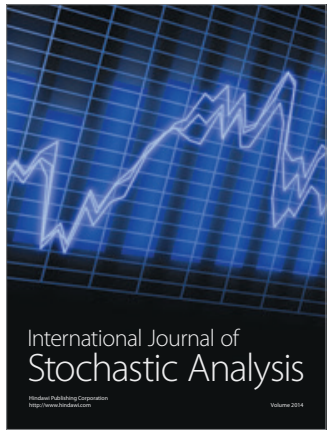

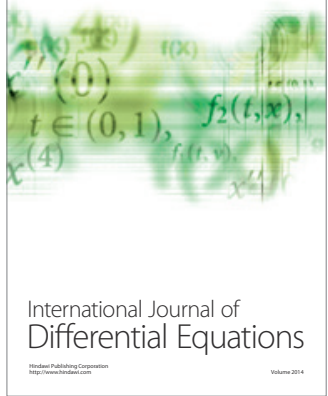
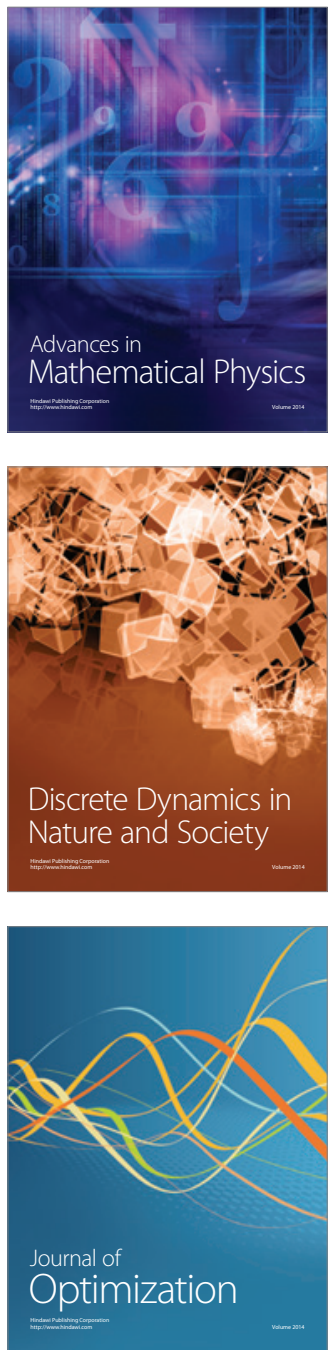